\title{
Factors Influencing the Prescription Behaviour of Doctors-An Insight for the Pharmaceutical CRM Strategy Formulation
}

Dr Gurpreet Kaur Chhabra

Assistant Professor, Management Education \& Research Institute, New Delhi, India.

Type of Work: Peer Reviewed.

DOl: http://dx.doi.org/10.21013/jmss.v15.n4.p6

\section{How to cite this paper:}

Chhabra, G.K. (2019). Factors Influencing the Prescription Behaviour of Doctors-An Insight for the Pharmaceutical CRM Strategy Formulation. IRA-International Journal of Management \& Social Sciences (ISSN 2455-2267), 15(4), 131-136. doi:http://dx.doi.org/10.21013/jmss.v15.n4.p6

\section{(C) Institute of Research Advances.}

\section{(cc) BY-NC}

This work is licensed under a Creative Commons Attribution-Non Commercial 4.0 International License subject to a proper citation to the publication source of the work.

Disclaimer: The scholarly papers as reviewed and published by the Institute of Research Advances (IRA) are the views and opinions of their respective authors and are not the views or opinions of the IRA. The IRA disclaims of any harm or loss caused due to the published content to any party.

Institute of Research Advances is an institutional publisher member of Publishers International Linking Association Inc. (PILA-CrossRef), USA. The institute is an institutional signatory to the Budapest Open Access Initiative, Hungary advocating the open-access of scientific and scholarly knowledge. The Institute is a registered content provider under Open Access Initiative Protocol for Metadata Harvesting (OAI-PMH).

The journal is indexed \& included in WorldCat Discovery Service (USA), CrossRef Metadata Search (USA), WorldCat (USA), OCLC (USA), Open J-Gate (India), EZB (Germany) Scilit (Switzerland), Airiti (China), Bielefeld Academic Search Engine (BASE) of Bielefeld University, Germany, PKP Index of Simon Fraser University, Canada. 


\begin{abstract}
Marketing has always been a major thrust area for the Pharmaceutical industry. Pharma firms usually bear a huge expenditure on marketing, especially sales efforts. Marketing in the Pharmaceutical industry is mainly done through personal selling techniques of promotion through MRs. Marketing of pharmaceutical products to doctors takes place through relationship marketing. Company representatives called medical reps meet the doctors in their specific geographic territory and promote their products to them. The doctors, in turn, prescribe the products to the patients who buy them from the chemist shop. Factors influencing doctor's prescription behaviour can be classified into three categories: Socio-cultural factors, Personal-Psychological factors, Pharma Marketing factors. Now a day's companies give a major thrust to the CRM aspect of their marketing strategies. Customer relationship management (CRM) is the strategic process of shaping the interaction between a company and its customers with the goal of maximizing current and lifetime value of customers for the company as well as maximizing, satisfaction for customers.CRM helps Pharma companies to generate revenue from shelf life by reaching more new prescribers (doctors) and patients (end customer) thereby keeping them loyal by increasing their well- being.
\end{abstract}

Key Words: Doctors, Medical Representatives (MRs), Pharmaceutical CRM, Pharmaceutical Marketing, Pharmaceutical CRM strategy.

\title{
Introduction
}

Foundation of CRM in the pharmaceutical industry is based on the prescribers (i.e. doctor) and sales force (MRs) relationship. Pharmaceutical firms spend a large amount of money on marketing communication directed towards physicians. Traditionally, salespeople in the pharmaceutical industry have been held accountable for short-term revenue generation to achieve higher sales volumes. However, due to the entry of more players in the market over the last decade, the matrix of success for pharmaceutical marketing has shifted perceptibly from customer revenue to customer profitability. Pharmaceutical companies rely on sales/medical representatives to deliver marketing messages to the Doctors. A sales/medical representative prepares a customer list (of doctors) in his or her sales territory and prepare their call plans for the day/week/month in accordance with this customer list. These representatives often do not enlist into the calling plan, those doctors who do not prescribe their products and devote extra time and effort on doctors who are most responsive to their products. If armed with the right information, a representative can call on doctors according to their likelihood of responding to specific brands, and can carry out detailing and sampling of specific brands resulting in higher customer profitability. Hence, they have to focus now on customer relationship management (CRM) and gain a complete understanding of their current and potential customers. Companies, which implement CRM, not only increase the efficiency of their sales and marketing efforts but also enhance their customer's experience and loyalty towards the pharmaceutical company. Some additional challenges that pharmaceutical companies in India are facing, includes: (i) Patients influencing doctors prescriptions (ii) Cost-effectiveness of the brand of drugs;(iii) Guarding against unethical dispensing of medicines at chemists level, which bypasses the physicians decision on specific brand of drugs (thereby defeating the whole purpose of the efforts done by the Pharma marketers). Critical decisions concerning strategy and resource allocation necessitate a detailed and accurate understanding of customers and the overall market. By using successful sales and service strategies, a pharmaceutical company could maximize customer profitability, improve marketing campaign effectiveness, and optimize Multi-channel interactions with customers, resulting in higher revenue and competitive advantage. The road map to profitability for pharmaceutical companies has been changing. Never before has the industry faced so many challenges at once. Harder-to-find blockbuster molecules, expiring patents, need to research for money-making drugs, pricing pressure from all players, and competition from new drugs, me-too drug and generics are all threatening industry profitability. In addition, issues such as: strict industry and government regulations, Drug Price Control Order (DPCO), increasing media and public scrutiny coupled with prescribing decision-makers inaccessibility (viz: doctors who have less time to spend with the Pharma firm's sales force) require more resources from companies just to maintain status- quo. All this has made it difficult to achieve the firm's profitability. The pharmaceutical landscape has, therefore, become more volatile and unpredictable. While these firms have to bear the soaring costs of Research and development, the purchasing power vested with few customers coupled with stringent regulations has made the promotional efforts more challenging. Today, the Pharma companies are forced to control spending on doctors' calls, in their efforts to drive profits.

There is a higher demand for pharmaceutical marketers to deliver bigger profits from increasingly smaller promotional budgets. These are a few critical decision areas, which, if addressed appropriately can help deliver 
better ROI. Therefore, many leading pharmaceutical companies are now looking for thorough access to customers data, which could enable them to improve the segmentation, targeting and positioning of specific brands. This process involves: I) identifying the key metrics and analytics needed to track trends in prescribing behaviour; II) designing, creating, implementing and executing marketing strategies that effectively measure ROI of core products;III) measuring customer satisfaction; IV) assessing the validity of ROI analysis, as compared to the competitors; and finally, V) using all the above information to determine the best allocation of promotional resources to identify the most appropriate segments to target for promotional investment optimization, accountability, and effectiveness.

Pharmaceutical marketers are asked today to deliver bigger profit margins from diminishing marketing budgets and to demonstrate accountability when making promotional investments. With a better understanding of ROI analysis, pharmaceutical companies can develop and implement more effective marketing communications, and thus enhance profitability. ROI will shape the future of a new commercial model for pharmaceutical companies and prepare product strategies for this shift in focus in order to understand how to utilize and map the potential life cycle of a product. Plan for strategic initiatives like upgradation of drug formulations and keying in individual roles can help shape up the direction of any brand of a pharmaceutical product. Last but not the least CRM helps Pharma companies to generate revenue from shelf life by reaching more new prescribers (doctors) and patients (end customer) thereby keeping them loyal by increasing their well- being. Finally, it is the Pharma CRM strategy that provides a base for new product innovations which provides a competitive advantage to these firms, to continue to thrive in the market and grow.

\section{Understanding Consumer Behaviour in the Pharmaceutical Industry- The Process of Prescription Generation}

The behavior is shown by people when they obtain, consume and dispose off products or services and which affects their choice of brands is known as consumer behavior. In the highly competitive world of today, the success of an organization is dependent on their ability to, not only satisfy customers but keep them delighted. Marketers are realizing the importance of customer retention. Majority of marketers today are focusing on gaining and maintaining customer loyalty instead of focusing only on customer creation. An insight into the consumer mind can help a firm build a competitive advantage. Study of human behavior is one of the most complex sciences. It is an inexact science; because it is almost impossible to predict human behavior. However, an attempt can be made to analyze and understand the reasons for a particular behavior shown by consumers, i.e. the factors that contribute to consumer behavior. In Pharmaceutical marketing, a firm has two types of customers: Primary customers; Doctors \& distribution channel members(mainly retailers) and Secondary Customers: Patients. However, the most important among these is the doctor. Without them, the sales cannot be generated. Therefore, understanding the behaviour of the doctors (prescribers) and identifying, understanding and evaluating the factors that influence their prescription behaviour forms the basis of CRM strategy of a Pharmafirm. Marketing of pharmaceutical products to doctors takes place through relationship marketing. Company representatives called medical reps meet the doctors in their specific geographic territory and promote their products to them. The doctors, in turn, prescribe the products to the patients who buy them from the chemist shop. The brands prescribed by the doctors depend upon their exposure to the different brand names present at any given time in the market, for a particular drug formulation. Their knowledge about the brands, in turn, depends upon the exposure given to them by the medical representatives of various pharmaceutical companies.

\section{Prescription Behaviour of Doctors- Diagnosis based Consultation approach}

Prescription behavior of doctors can be defined as the process of prescribing a particular brand or formulation of a medicine or drug for the treatment of a particular ailment by a doctor, to a patient. When a patient explains about his /her health problem the doctor, first of all, asks about the symptoms and based on this information tries to diagnose the disease and then prescribes the drug/drug formulation that is suitable for that particular ailment. While the doctor decides about the drug to be prescribed at the same time, he/she also makes the decision about the brand of the drug formulation that is to be prescribed to the patient. While in western countries doctors prescribe formulation and not the brand and the choice of brand to a great extent depends upon the retailer (chemist).In India however, the scenario is a brand-based prescription from the doctor.

While deciding upon the molecule and then the brand of medicine to be prescribed to the patient, a doctor has to 
consider a lot of social perspectives apart from the general medical aspects. One of the major social perspectives is that of the price of the medicine. In a country like India where literacy rate is low and patients trust doctors as God, doctor, in turn, has to live up to the expectations and trust shown by the patients in them and therefore while prescribing a medicine, a doctor often consider a lot of parameters and many factors influence the prescription given by him/her. Patients often prefer or insist on the brand of medicine prescribed by the doctor while they go to buy the same from the chemist counter. Substitution if possible, may take place at the chemist counter that too in some cases when the specific brand is not available or same formulation is available at low price in the form of generic drugs.

\section{Factors Influencing the Prescription Behaviour of Doctors}

Although there are many documented as well as many undocumented factors that influence the prescription behaviour of doctors. Many types of research have been carried out around the world to identify and study the factors that define, determine and have an influence on the prescription behaviour of doctors. However, there has not been much agreement on the parameters that influence the choice of prescribing a particular brand by a doctor. Different researchers have identified and studied different variables as the factors that influence Doctor's prescription habits. Broadly these factors can be classified into three categories: Socio-cultural factors, PersonalPsychological factors, Pharma Marketing factors.

Socio-cultural factors mainly include the role and influence of culture, social class, and reference groups. An important point to understand over here is that culture is determined by the country, category and speciality of the doctor whereas the social class of the patient to whom the medicine is being prescribed influences the choice criteria of the brand being prescribed. Among all these factors, the reference group is considered to be the most important factor influencing not only the choice of the molecule/drug salt being prescribed but also the brand to be prescribed. Personal-Psychological factors play the most crucial role because these factors vary from individual to individual. The factors include the role and influence of age, gender, personality, self-concept, learning, perception, motivation, attitudes, beliefs, etc. Many types of research indicate that the age and gender of the doctor do influence his/her choice criteria while others indicate that it has no impact on the choice of brand prescribed. Among these factors' personality, learning, motivation, attitudes, and beliefs are the factors that seem to have the maximum influence on the choice criteria of doctors. While personality is a factor that has not been researched about, its influence on prescription behaviour of a doctor but learning, motivation, attitude, and beliefs are formed, shaped and modified by the marketing strategies adopted by the pharmaceutical companies. Marketing factors such as detailing visits by MR, distribution of free samples, promotion through special campaigns, authentication of drug efficacy through clinical trials, sponsorships for CMEs, conferences, Medical updates, and related seminars and value-added gifts on special occasions like festivals, birthdays, anniversaries, etc.

Influence of Opinion Leaders on Prescription Behaviour of Doctors- An opinion leader is a well-known individual that has an influence on the public opinion on the topic or the matter for which he/she is known. In the field of pharmaceuticals, doctors having prolific practice, good clientele and very high success rate in their specific domain are called opinion leaders. They can act as an opinion leader in their respective local geographical areas or with time they may become a national or international figure. Pharma companies usually invite them to deliver a speech/lecture/share their views on the topic of mutual interest, which the company feels, may influence the behaviour of other doctors in their own field. Along with this, the influence of Pharma CRM strategies in shaping and influencing the learning \& motivation of the doctor and how collectively these factors form the attitude and beliefs of the doctors towards the selected Pharma firms and the brands promoted by them have been the theme of this research study. These factors try to influence the two parameters of the prescription:

- A molecule of the drug(product choice) to be prescribed- there are various drug molecules preferred in a particular indication(ailment/disease) and the doctor has to decide which among them to be selected for a prescription.

- Choice of medicine(brand choice) to be prescribed- one type of drug molecule is being manufactured/promoted by multiple pharma companies. Choosing a brand of a particular pharmaceutical company has to be decided by the doctor.

Influence of Motives behind prescribing a particular product- A motive is a purpose for which a person buys the goods or avails a service. These motives could be a mere thought erupted suddenly, a sudden urge, a feeling 
experienced, an urgent desire or an expressed emotion which results in the form of reaction from the buyer, to purchase. In pharmaceuticals motives can be studied at two levels:

A) Doctor's buying(prescribing) motive

B) Patient's Buying motive

Doctor's buying motive cannot be explicitly explained because there are numerous factors that influence the buying behaviour of doctors and in this research study the underlying factors influencing doctor's buying behaviour have been studied at length.

Patient's buying motive is surfaced when he/she comes to visit a doctor when he/she experiences a state of physical or mental illness. Whatever is prescribed to him/her, the patient has to buy the same from the chemist. In certain cases, the price can act as a limiting factor and therefore the patient may switch brand or may request the doctor to prescribe some low-priced brand.

\section{Methods Adopted by Pharma Companies to Influence the Prescription Behaviour of Doctors}

As discussed, earlier marketing in the Pharmaceutical industry is mainly done through personal selling techniques of promotion through MRs. In each visit, an MR presents selected brands from the brand portfolio of his/her company. Choice of the brand for presenting to a doctor depends upon the speciality of the doctor, patient profile, prescription behaviour of the doctor and doctor's attitude towards salesperson or the company that he/she represents. A detailing visit generally lasts for 5-7 minutes or at times even less. The MR shares information about the composition of the drug, its therapeutic value, proper dosage, potential side effects, etc. with the doctor during this detailing visit (Sulliveen, Bridwell \&Kulukarslam, 1994). On average, it requires 1-10 more visits called reminder or follow up visits by the MR to generate one new prescription (Kante,2010). Further, the MR needs around 20-73 additional free samples to induce one new prescription (Kante, 2010). Research indicates that doctors irrespective of their speciality, are not easy targets who can be easily persuaded by MRs. In fact, they are hard nuts to crack due to the intense pressure of pharma promotional activities on their prescription behaviour (Tracy \& Smith, 1994). However certain studies indicate that gynaecologists especially female doctors are receptive towards the detailing \& information shared by the MRs (Kasliwal, 2013). Majority of studies explain the reason for the limited effect on doctor's prescription behaviour. According to these studies' other tools of Pharmaceutical marketing, in order of preference or the ability of their influence, are CMEs or other knowledge updating sponsorships>Scientific information updates $>$ Promotion Inputs>Reputation of the company (Kohatkar\& Inamdar,2012). Researches across specialities of doctors revealed that they rely on other sources of information such as journals, research papers, advice from Key opinion leaders of their respective speciality and of course their own training and experience affects their prescription behaviour and they view these sources as far more reliable and trustworthy in comparison to the MRs detailing information(Harrington,2002).

\section{CRM Strategy the Tool for Influencing the Prescription Choice Criteria of the Doctors}

CRM is a business strategy to select and manage valuable customer relationships. It requires a customer-centric business philosophy and culture to support effective marketing, sales, and service processes. CRM application can enable effective CRM provided that an enterprise has the right leadership strategy and culture(Thompson,2005). In terms of strategic management, CRM is not just a strategic concept, rather it a concept that is built upon a clear-cut understanding of the processes and information systems underlying these concepts. The central activity in CRM strategy is exploiting customer insight and information to create profitable customer relationships. According to Buttle (2003), Marketing is no longer about developing, selling and delivering products. It is progressively more concerned about the development and maintenance of mutually satisfying long-term relationships with customer'. According to him enduring relationships with customers cannot be duplicated by competitors and therefore provide for a unique and sustained competitive advantage. This view is further supported and augmented by Tzokas\& Saran. According to them, these relationships developed with the customers eventually become the core competencies of the organization which is non-imitable by other firms. A major aspect of the promotional strategy adopted by the Pharma firms in India has been, personal selling through medical representatives (MRs). However, from the last few years particularly after the introduction of the product patent regime in 2005, market scenarios started changing drastically. To add to the trouble of Pharma marketers, in a development in July 2012 the Indian Pharma companies agreed in principle to enforce a code that will restrict them from offering gifts or other SOPS to doctors. This has resulted in changes in the level, intensity, and parameters of competition. MRs who earlier acted as the backbone for 
the Indian Pharma sector, are finding it very hard to compete, and to make every interaction with doctors, count. Today the Pharma companies that leverage technology solutions such as ERP, CRM, mobility solutions, only have the scope of survival in the future. Pharmaceutical companies are gradually realizing that they can no longer afford to be only product or market focused. They have to focus now on relationship marketing or customer relationship management (CRM) in order to gain a complete understanding of their current and potential customers. By implementing CRM, not only the efficiency of sales and marketing efforts increase but also the customer's experience and loyalty towards the pharmaceutical company is enhanced.

\section{Conclusion}

In a nutshell, CRM is all about identifying, satisfying, retaining and maximizing the value of a company's best customers. Its time not that the Indian pharmaceutical market has to re-work upon its ground level of understanding consumer(doctor) behaviour. Doctors who are the actual customers of these firms are also human beings and therefore their purchase/prescription behaviour is also influenced by various factors. Some of which are internal while some are external. The base of any marketing strategy is a thorough understanding of consumer behaviour. A proper understanding of factors that influence prescription behaviour of doctors, can help a pharmaceutical marketer design suitable CRM strategy based on proper segmentation, targeting and positioning approach. All in all, it can be concluded that prescribing the behaviour of doctors is a complex activity involving the interplay of a number of factors which varies from marketing, relationship \&CRM strategies adopted by Pharmaceutical companies to medical \& psychosocial factors. If the CRM strategists have a thorough insight into these factors, they shall be able to design the right CRM strategy for each category of doctors and with this they will not only save the time, efforts and money spent by the company on promotion of their products but will also improve the effectiveness and efficiency of their medical representatives.

\section{References:}

[1]. Buttle, F.(2003).Customer relationship management Concepts and tools.1st ed. Oxford: Butterworth- Heinemann Publishing House, New York, USA.

[2]. Kante\&Rajesh, U. (2010). Self-Medication, Doctor, and Marketing of OTC Products. Asian journal of management research,229-238.

[3]. Kasliwal, N.(2013).A Study of Psycho-Social Factors on Doctors Prescribing Behaviour - An Empirical Study in India. IOSR Journal of Business and Management,13(2),5-10.

[4]. Kolhatkar, J.Malhar, Inamdar \&S.Irfan. (2012).Doctor's Expectations from Pharmaceutical Companies: Which will Influence their Prescription Behavior. International Journal of Business management tomorrow, 2(1), 1-6.

[5]. Thompson, B.(2005).What is CRM? Retrieved fromhttp://crmguru.custhelp.com/cgi/

[6]. Thomas P., Rainer A. (2001) Customer Relationship Management in the Pharmaceutical Industry, Proceedings of the 34th Hawaii International Conference on System Sciences - 2001. 